\title{
A PROBLEM ON THE BLOCH NORM OF FUNCTIONS IN DOOB'S CLASS
}

\author{
J. S. HWANG ${ }^{1}$
}

ABSTRACT. Let $\Delta$ denote the unit disc and $\partial \Delta$ denote the unit circle both in the complex plane. Define the Doob's class $D(\rho), 0<\rho<2 \pi$, as all holomorphic functions on $\Delta$ satisfying (1) $f(0)=0$, and (2) for some arc $\Gamma_{f} \subseteq \partial \Delta$ with arclength $\rho$, for all $p \in \Gamma, \underline{\lim }_{z \rightarrow p}|f(z)| \geq 1$.

Recently the author and Rung [6] proved a conjecture of Doob made in 1935 by showing that the norm

$$
\|f\|=\sup _{z \in \Delta}\left(1-|z|^{2}\right)\left|f^{\prime}(z)\right| \geq \frac{2 \sin \theta(\rho)}{e \theta(\rho)}, \quad 0 \leq \theta(\rho) \leq \pi-\rho / 2 .
$$

We then conjecture that the result should be true if the arc $\Gamma_{f}$ is replaced by a finite union of arcs whose total length is at least $\rho$. In this paper, we answer this problem. It turns out to be surprising that the answer depends on the connectivity of the union, namely, the answer is no for the disconnected case, but yes for the connected one.

1. Introduction. Earlier in 1935 [2], J. L. Doob introduced a family $D(\rho)$ of holomorphic functions in the unit disk $\Delta$, whose boundary we denote by $\partial \Delta$. For any fixed $0<\rho<2 \pi, D(\rho)$ is the family of all holomorphic functions in $\Delta$ which satisfy (i) $f(0)=0$, and (ii) $\underline{\lim }_{z \rightarrow p}|f(z)| \geq 1$, for all $p$ lying on some arc $\Gamma_{f} \subset \partial \Delta$ whose arc length $\left|\Gamma_{f}\right| \geq \rho$. Doob posed the question as to whether the set of Bloch norms $\|f\|=\sup _{z \in \Delta}\left(1-|z|^{2}\right)\left|f^{\prime}(z)\right|, f \in D(\rho)$, has a positive lower bound. The author and Rung showed in [5] that for each $f \in D(\rho)$ there exists a point $z_{f} \in \Delta$ at which

$$
\left(1-\left|z_{f}\right|^{2}\right)\left|f^{\prime}\left(z_{f}\right)\right| \geq \frac{2 \sin (\pi-\rho / 2)}{e(\pi-\rho / 2)} .
$$

Recently, in [6] the same authors improved the right side of (1) by replacing the quantity $\pi-\rho / 2$ with a value $\theta(\rho)$ which lies between 0 and $\pi-\rho / 2$. At the end of [6], it was conjectured that this result should be true if the arc $\Gamma_{f}$ is replaced by the finite union of arcs whose total length is at least $\rho$ in the above condition (ii). In this paper, we shall answer this conjecture in two ways depending on whether the closure of the finite union of arcs is connected or not. The answer turns out to be no if it is not connected, but almost yes if it is connected.

THEOREM 1. Let $\varepsilon>0$ and $\delta>0$ be given, then there is a function $f \in$ $D(2 \pi-\varepsilon)$, where $\Gamma_{f}$ is the finite union of disjoint arcs whose total length is at least $2 \pi-\varepsilon$, and the Bloch norm $\|f\|<\delta$.

Received by the editors June 29, 1984 and, in revised form, January 25, 1985.

1980 Mathematics Subject Classification. Primary 30C80; Secondary 30D40.

Key words and phrases. Bloch norm, Doob's class, maximum principle.

${ }^{1}$ I am indebted to the referee for providing the proof of Theorem 2 which is much shorter than my original proof. 
THEOREM 2. Let $f$ be holomorphic in $\Delta$ satisfying conditions (i) and (ii) except at an interior point $q$ of $\Gamma_{f}$, i.e. (ii) does not hold at $q$, then we have $\|f\| \geq 2 / e$ provided one of the following three conditions holds: (2) $f$ has a sequence of zeros tending to $q$ tangentially,

(3) $f$ has radial limit 0 at $q$,

(4) $f$ omits 0 in a neighborhood of $q$ relative to $\Delta$.

Notice that in each case of Theorem 2 we obtain (1).

2. Proof of Theorem 1. Let $S$ be a set of finitely many points in $\Delta$ such that $S$ does not contain the origin. Let $f$ be the conformal mapping from $\Delta$ onto the universal covering surface of $\Delta-S$ such that $f(0)=0$. If $f$ has radial limit on the radius $\left[0, e^{i \theta}\right)$, then we denote the radial limit by $f\left(e^{i \theta}\right)$. It follows from a theorem of Seidel $\left[8\right.$, Theorem 10] that the set $T=\left\{e^{i}: f\left(e^{i \theta}\right) \in S\right\}$ is a perfect set on $\partial \Delta$. Moreover, the radial limit $f\left(e^{i \theta}\right)$ is of modulus one for any $e^{i \theta} \notin T$. Clearly, this set $T$ is of measure zero due to a theorem of Riesz, see [1, Theorem 2.5]. Let $\varepsilon>0$ be given, then there is a set $\Gamma_{f} \subset \partial \Delta$ which is the finite union of disjoint arcs contained in $\partial \Delta-T$ such that the total length $\Gamma_{f}$ is at least $2 \pi-\varepsilon$. It follows that

$$
\left|f\left(e^{i \theta}\right)\right|=1, \quad \text { for each } e^{i \theta} \in \Gamma_{f},
$$

and therefore condition (ii) is easily seen to be satisfied.

According to a theorem of Bloch, see Hille [4, Theorem 17.7.1], we know that the range of $f$ over $\Delta$ will cover the interior of some circle of radius $B\|f\|$, where $B$ is a constant. Let $\delta>0$ be given, then the set $S$ can be chosen so that the complement $\Delta-S$ contains no disk of radius $B \delta$. This implies that $\|f\|<\delta$ and the proof is complete.

3. Proof of Theorem 2. Assume $\|f\|<2 / e$, and choose an $\alpha$ small, $0<\alpha<\pi$, so that $\|f\|<2 \sin \alpha /(e \alpha)$. As in [5, p. 232], we define the lens shaped domain by

$$
L(\alpha, \Gamma)=\{z: \omega(z, \Gamma, \Delta)>\alpha / \pi\},
$$

where $\omega(z, \Gamma, \Delta)$ is harmonic in $\Delta$ and assumes the value 1 and 0 on $\Gamma$ and $\partial \Delta-\Gamma$, respectively. Then by the same argument as in the proof of Theorem 1 of $[\mathbf{5}]$ which is based upon the result of Dragosh and Rung [3], we have

$$
|f(z)| \geq 1 / e \text { in } L\left(\alpha, \Gamma_{1}\right) \cup L\left(\alpha, \Gamma_{2}\right),
$$

where $\Gamma_{1}$ and $\Gamma_{2}$ are two adjacent arcs separated by the point $q$, and (ii) holds on each $\Gamma_{i}, i=1,2$. Thus condition (2) of Theorem 2 cannot hold, nor can condition (3) because $f$ is normal and so would have nontangential limit 0 on parts of $L\left(\alpha, \Gamma_{i}\right)$.

Finally, let $R$ be a subregion of $\Delta-\left(L\left(\alpha, \Gamma_{1}\right) \cup L\left(\alpha, \Gamma_{2}\right)\right)$ whose boundary meets $\partial \Delta$ only at $q$. If $f$ omits 0 in $R$, and we assume $1 / f$ is unbounded in $R$, then by the Gross-Iversen Theorem [1, Theorem 5.8], there is an asymptotic path ending at $q$ on which $1 / f \rightarrow \infty$ and we have condition (3). If $1 / f$ is bounded in $R$ then $1 / f$ is bounded above in a full neighborhood of $q$ and so $f$ satisfies (ii) at $q$, contradicting the hypothesis. This completes the proof.

We note that the assertion of Theorem 2 with regard to condition (3) also follows from [7, Theorem 1]. 


\section{REFERENCES}

1. E. F. Collingwood and A. J. Lohwater, The theory of cluster sets, Cambridge Univ. Press, London and New York, 1966.

2. J. L. Doob, The ranges of analytic functions, Ann. of Math. (2) 36 (1935), 117-126.

3. S. Dragosh and D. C. Rung, Normal functions bounded on arcs and a proof of the Gross cluster-value theorem, Hiroshima Math. J. 9 (1979), 303-312.

4. E. Hille, Analytic function theory, vol. II, Ginn, Boston, Mass., 1962.

5. J. S. Hwang and D. C. Rung, Proof of a conjecture of Doob, Proc. Amer. Math. Soc. 75 (1979), 231-234.

6. __ An improved estimate for the Bloch norm of functions in Doob's class, Proc. Amer. Math. Soc. 80 (1980), 406-410.

7. J. S. Hwang, On an extremal property of Doob's class, Trans. Amer. Math. Soc. 252 (1979), 393-398.

8. W. Seidel, On the distribution of values of bounded analytic functions, Trans. Amer. Math. Soc. 36 (1934), 201-226.

Institue of Mathematics, Academia Sinica, Taipei, Taiwan, China 